\title{
Reduction of malaria
} prevalence after introduction of artemisinin-combination-therapy in Mbeya Region, Tanzania: results from a cohort study with 6773 participants

Guenter Froeschl $\left.\right|^{1,2^{*}+}$ D , Elmar Saathoff ${ }^{1,2+}$, Inge Kroidl ${ }^{1,2}$, Nicole Berens-Riha $^{1,2}$, Petra Clowes ${ }^{1,3}$, Leonard Maboko ${ }^{3}$, Weston Assisya ${ }^{3}$, Wolfram Mwalongo ${ }^{3}$, Martina Gerhardt ${ }^{3}$, Elias Nyanda Ntinginya ${ }^{3}$ and Michael Hoelscher ${ }^{1,2}$

\begin{abstract}
Background: A marked decline in malaria morbidity and mortality has been reported after the introduction of artemisinin-based combination therapy (ACT) in high malaria prevalence countries in Africa. Data on the impact of ACT and on the prevalence of malaria has so far been scarce for Southwest Tanzania.

Methods: Between 2005 and 2011, a large general population cohort in the Mbeya Region in the south-west of Tanzania has been surveyed within the EMINI-study (Evaluation and Monitoring of the Impact of New Interventions). Participants were examined once per year, including rapid diagnostic testing for malaria. ACT was introduced in the region according to national guidelines in the time period 2006/2007, replacing sulfadoxine/pyrimethamine as firstline therapy. In four study sites, 6773 individuals who participated in the first two of three consecutive survey visits in the period from 2006 to 2009 were included in this analysis. The prevalence of Plasmodium infection prior to and after the introduction of ACT was compared by logistic regression, with consideration of climatic variability, age, sex, socioeconomic status and bed net use as potential confounders.
\end{abstract}

Results: A significant reduction over time in the prevalence of Plasmodium falciparum infection from 2.5 to $0.3 \%$ was shown across the four study sites. The decline was not explained by other factors included in the analysis, therefore, the decline over time most likely reflects the impact of introduction of ACT in the study area.

Conclusions: The longitudinal study showed a significant and relevant decline in the prevalence of $P$. falciparum infection after introduction of ACT, which could not be explained by potential confounders. The data suggests that artemisinin-based combinations are not only an effective instrument for reduction of immediate morbidity and mortality, but also for reduction of transmission rates.

Keywords: Artemisinin-based combination therapy, Cohort study, Normalized Difference Vegetation Index, Bed net, Malaria, Plasmodium falciparum, Prevalence, Rapid diagnostic test, Mbeya Region, Tanzania

\footnotetext{
*Correspondence: froeschl@|rz.uni-muenchen.de

${ }^{\dagger}$ Guenter Froeschl and Elmar Saathoff contributed equally to this work

${ }^{1}$ Division of Infectious Diseases and Tropical Medicine, University

Hospital, LMU Munich, Leopoldstr. 5, 80802 Munich, Germany

Full list of author information is available at the end of the article
} 


\section{Background}

According to the World Malaria Report 2016, to date still more than 210 million persons suffer from malaria infections every year, with 429,000 malaria deaths in 2015 worldwide. The major burden of malaria still lies within Africa with more than 190 million cases and 394,000 deaths annually. Almost all malaria cases in sub-Saharan Africa are caused by the species Plasmodium falciparum. This high mortality in Africa is aggravated by the high proportion of deaths in the under- 5 age-group, which account for about $90 \%$ of all malaria related deaths, more than in any other global region.

The East-African country Tanzania has a total population of 45 million, of whom $44 \%$ are under the age of 15 , and $15 \%$ under the age of 5 years. A Tanzanian has a combined life expectancy at birth of 56 years, the total fertility rate is 6.3 children per woman, the annual population growth rate is $2.7 \%$ [1]. The per capita gross domestic product is US\$ 652 and the Human Development Index of 0.521 is ranking 151st out of 186 countries [2]. According to World Health Organization (WHO) data on Tanzania, malaria causes $10 \%$ of all deaths in the under-5-age-group, while the overall under-5-mortality in 2012 was 52 deaths per 1000 live births [3]. The total malaria deaths for Tanzania for all age groups was more than 20,000 in 2012, and it may be assumed that the large majority of these deaths are again found in children below 5 years. In a prevalence study conducted in Central Tanzania in 2015, 22.5\% of participants between 1 and 10 years of age and $26.8 \%$ between 11 and 20 years were found rapid diagnostic test (RDT) positive for malaria [4]. Malaria still is a major public health concern in Tanzania and among the top five causes of death for children under 5 years of age [3].

In order to tackle this problem, particularly in countries with a high malaria burden, which are at the same time low-resource-countries, global stakeholders, such as the WHO, the Global Fund to Fight AIDS, Tuberculosis and Malaria and the Gates Foundation have engaged in promoting aims of malaria reduction that have been set forth in the Millennium Development Goals. Consecutively the World Health Assembly and the Roll Back Malaria initiative have envisaged a reduction by $75 \%$ in malaria disease burden between the years 2000 and 2015. Interventions include vector and exposure control through indoor residual spraying (IRS), distribution of insecticide-treated bed nets (ITN), introduction of RDT for differentiation between true malaria cases and nonmalarial febrile illnesses, and introduction of artemisininbased combination therapy (ACT) as first-line treatment [5]. Subsequently, numerous high malaria burden countries such as Zambia or Tanzania have reported declines in malaria incidence [6-8]. However, data on malaria prevalence remains patchy, and solid monitoring mechanisms are absent.

Even after the large-scale implementation of rapid diagnostic testing and ACT in Tanzania, high prevalences of parasitaemia in many regions imply a persistent risk of transmission to vulnerable populations, such as infants. In a study from Northern Tanzania in 2012/2013, thus well after the introduction of ACT and RDTs, a prevalence of plasmodium infection of 12.8 and $11.0 \%$ were detected in pregnant women and in infants respectively, regardless of presence of symptoms [9]. Another study from Northern Tanzania that was trying to identify Dengue and Chikungunya virus infections as causes of febrile illness employed the WHO case definition criteria for Dengue and Chikungunya as criteria for recruitment of febrile patients. Still $8.8 \%$ of these participants were infected with malaria [10]. The adequate use of anti-malarials, or even an anti-malarial stewardship, as the key factor for prevention of drug resistance remains a challenge, while uncontrolled distribution of valuable treatment options, such as ACT remains common practice [11].

In this study, the prevalence of malaria RDT positivity before and after the introduction of ACT was compared in order to estimate the effect of ACT introduction on malaria in the study population.

\section{Methods}

\section{Study setting, study design and population}

The Mbeya Region with a population of 2.7 million is located in the Southwest of Tanzania, a highland area with mountains ranging up to $3000 \mathrm{~m}$ in altitude [1]. The lowest point is the shore of Lake Malawi at about $500 \mathrm{~m}$ above sea level. The climate at lower elevations is subtropical with a rainy season from December to April. The average annual rainfall for Mbeya (years 1991-2015) is $883 \mathrm{~mm}$ [12]. Night-time temperatures in the dry season can drop below $10{ }^{\circ} \mathrm{C}$ at higher altitudes.

This study is an observational, longitudinal and closed cohort study, based on the EMINI (Establishment of the infrastructure for the Evaluation and Monitoring of the Impact of New Interventions) cohort, established since May 2006 by the Tanzanian National Institute of Medical Research-Mbeya Medical Research Centre (NIMRMMRC) in collaboration with the Division of Infectious Diseases and Tropical Medicine, University Hospital, LMU Munich. The design follows a pre- and post-intervention structure, where the intervention was a switch of government first-line malaria treatment from SP to ACT in the whole population. In order to properly represent the regional diversity in terms of geography, urbanization and living conditions, nine study sites in the Mbeya Region were purposively selected for participation in the 
EMINI cohort, covering characteristics, such as urbanization and different levels of altitude. In preparation for EMINI, a complete population census was performed in all nine sites. In order to get a geographically balanced sample and avoid random spatial clustering, each site was then subdivided into 6-10 areas, from each of which 10\% of households were randomly selected for participation in the EMINI cohort. All household members of all ages from these selected households were then invited for participation, resulting in a cohort of more than 18,000 individuals in the nine EMINI sites. Because the introduction of ACT coincided with the set-up of the cohort in five of the nine sites, no consistent pre-ACT baseline data is available for these sites. Hence, only the remaining four sites, where ACT was introduced after completion of the first baseline visit were included in this analysis, comprising 7957 individuals. Out of these, 1184 individuals were not present at the second household visit, resulting in 6773 individuals who were included in our analyses.
The included four sites are the rural areas of Igurusi (1190 m, population 19,796), Mlowo (1590 m, population 21,307), Santylia (2020 m, population 21,149) and Isongole (2040 m, population 20,360) (Fig. 1). The above reference population numbers were retrieved from an initial population census before the start of the actual EMINI cohort study, while the indicated altitudes of the sites are the mean altitudes of household positions in each site, which were retrieved from the global elevation model (version 2.1) of the US National Aeronautics and Space Administration (NASA) Shuttle Radar Topography Mission (SRTM) [13, 14].

The analysis includes data from three annual rounds of household visits which took place between May 2006 and June 2007 (visit 1), July 2007 and July 2008 (visit 2), and July 2008 and June 2009 (visit 3). During each round, the same sites and households were visited at about the same time of the year.

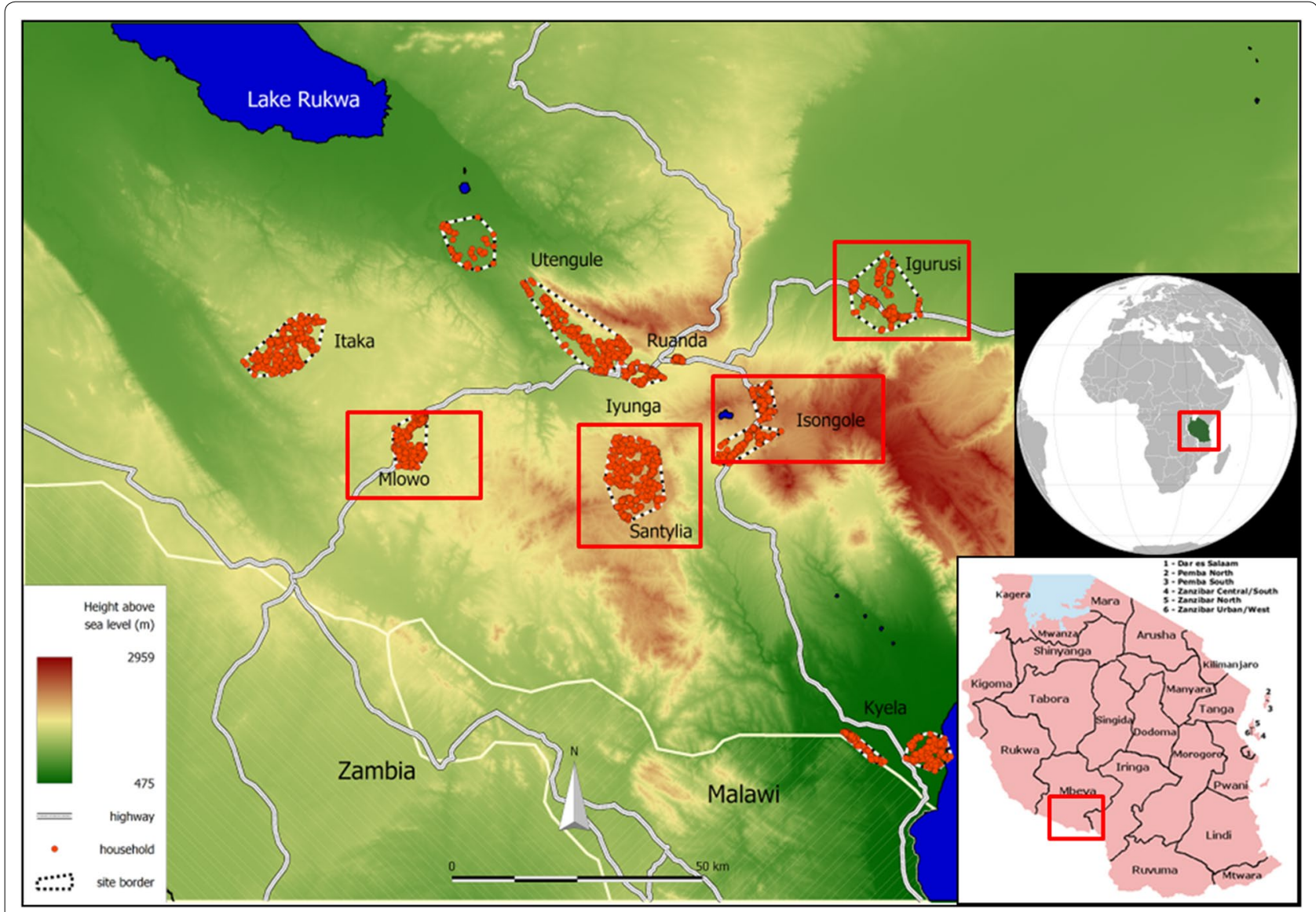

Fig. 1 Map of study sites in the Mbeya Region. The larger image shows the location of the nine EMINI study sites in the Mbeya Region with red dots indicating household positions. Red rectangles indicate the four sites included in our analysis. The inserted images show the locations of Mbeya Region in South-West Tanzania, and of Tanzania in East Africa 


\section{Variables and instruments}

Plasmodium falciparum infection was tested using the ICT Malaria Combo Cassette Test (ICT Diagnostics, Cape Town, South Africa). This RDT is an immunochromatography test with antibodies against histidine-rich protein 2 (HRP2), which is species-specific for $P$. falciparum, in combination with antibodies against aldolase which is detected in P. falciparum, Plasmodium malariae, Plasmodium ovale and Plasmodium vivax infections. For this study $P$. falciparum positivity was defined as any RDT result with both a positive control line and a positive HRP2 line, regardless of the result of the aldolase line. The test was performed on every participant at each household visit, regardless of clinical signs of malaria. Participants with a positive result were offered immediate treatment with ACT. Since ACT was introduced in the entire region, no control group continuing to be exposed to the previous first-line treatment could be included in the analysis. RDTs and immediate treatment provision, restricted to confirmed cases only, were already in place in all sites prior to the study initiation.

Information on personal and household characteristics (age, sex, and indicators of socio-economic status) was collected using questionnaire based face-to-face interviews, which were held in Kiswahili. Bed net ownership and housing quality was ascertained by visual inspection. A socio-economic status (SES) score was constructed as a composite and standardized variable based on a principal component analysis of several household assets and housing quality $[15,16]$.

The geographical position of each participating household was determined using handheld GPS (Global Positioning System) receivers. Vegetation characteristics were quantified using annual averages of the Normalized Difference Vegetation Index (NDVI), which is based on satellite measurements as provided by the US National Aeronautics and Space Administration (NASA). Differences in light absorption spectra measured by spectral cameras in satellites allow the assessment of vegetation density, which in turn allows conclusions on previous floral growth conditions, such as humidity and rainfall profile. This vegetation data is available from the NASA Moderate-Resolution Imaging Spectroradiometer (MODIS) Terra Mission [17, 18]. NASA gives as an approximate interpretation a range for the NDVI from 0.2 to 0.3 for shrub and grassland, while values between 0.6 and 0.8 indicate temperate and tropical rainforests. Elevation data were retrieved from the NASA Shuttle Radar Topography Mission (SRTM) global digital elevation model, version 2.1 [13]. NDVI and elevation data were averaged for a buffer area of $1000 \mathrm{~m}$ radius around each household position to characterize the situation at household level.
The performed analyses are based on data before (visit 1 ) and after (visit 2) introduction of ACT. In order to corroborate the sustainability of reduction in $P$. falciparum infections, prevalences for visit 3 are also reported.

\section{Statistical analysis}

All interview and medical examination data were collected using hand-held personal digital assistance devices and then imported into a Microsoft Access (Microsoft Corporation, Redmond, USA) database. Laboratory results were documented on paper forms, double entered into a Microsoft Access database and compared and corrected for data entry errors. Data analysis was performed using Stata version 14.2 (StataCorp, College Station, TX, USA). The univariable description of the cohort uses proportions to summarize categorical variables. Continuous variables are summarized by their median and interquartile range, since none of them was normally distributed according to the Shapiro-Francia test.

Two mixed effects multivariable logistic regression models with random effects for study site and household to adjust for clustering were constructed. The first model examines the effects of various factors on the baseline prevalence of malaria RDT positivity, with the RDT result of visit 1 as the outcome and sex, age, bed net ownership, SES and NDVI as covariates. The second model targets the change in RDT positivity from visit 1 to visit 2 . Change is dichotomously defined as a change from positive in visit 1 to negative in visit 2 versus all other scenarios combined (positive at both visits, negative at both visits, negative in visit 1 to positive in visit 2 ). In this model the independent variables sex, age, change in bed net ownership, change in SES and change in NDVI from visit 1 to visit 2 were included. In 4 out of 6773 participants data on SES was missing, therefore the models were run on 6769 participants.

\section{Ethical considerations}

This study follows the principles of the Helsinki Declaration. All participants had signed an informed consent form prior to inclusion. For all minors below the age of 18 years their legal guardians signed the informed consent form and children between 12 and 18 years additionally signed an assent form. Ethics approval of the cohort study within the EMINI framework was obtained from the Tanzanian National Institute for Medical Research.

\section{Results}

\section{Cohort background characteristics}

The lower lying sites of Igurusi and Mlowo are characterized by lower NDVI values than Santylia and Isongole. The numbers of inhabitants of the four included sites according to the EMINI census data, the mean altitude 
and the mean vegetation-index of households at the respective sites are detailed in Table 1.

Table 2 describes the study population of the four sites. Female participants were significantly older than male participants (median 17.4 years for females, 14.4 years for males, Mann-Whitney $\mathrm{p}<0.0001)$. In Igurusi and Mlowo average SES was higher than in Santylia and Isongole.

\section{Vegetation index}

The four sites showed very different NDVI distributions, which corresponds to the varying physiognomy of the locations. Most NDVI values were in the range between 0.3 and 0.6 , and thus between grassland and temperate/ tropical forest according to NASA [19]. This reflects the savannah landscape that is mostly found in the populated areas of Mbeya Region. Mean relative NDVI change between visit 1 and visit 2 for each site ranged from -2.55 to $+2.90 \%$ (Table 1 ). While NDVI decreased in Igurusi $(-0.50 \%, 95 \%$ confidence interval: -0.61 to $-0.39 \%)$, Mlowo $(-2.55,-2.60$ to $-2.49 \%)$ and Santylia
( $-0.21,-0.44$ to $0.01 \%)$, Isongole showed an increase in NDVI (2.90; 2.71 to $3.09 \%)$. However, overall these changes can be considered as minimal.

\section{Plasmodium falciparum prevalence}

Marked differences between the different study sites regarding baseline prevalences of $P$. falciparum infection were found (Table 2). As expected, lower prevalences already at baseline could be shown for the two locations at higher altitudes $(0.4 \%$ in Santylia and $0.9 \%$ in Isongole) compared to the lower lying sites (7.3\% in Igurusi and $3.1 \%$ in Mlowo). For all four included study sites, a decrease of $P$. falciparum infection prevalence after introduction of ACT was observed regardless of altitude, which most prominently occurred between the baseline visit and the first follow-up visit. Figure 2 highlights the crude decline of prevalence of positive RDT results for all sites over time.

Across all four included sites, 160 out of 6773 individuals were RDT positive at visit 1 and RDT negative at visit

Table 1 Study site characteristics

\begin{tabular}{|c|c|c|c|c|c|}
\hline Site & Mean altitude (m) & Total population & Number of participants & Mean NDVI at visit 1 & $\begin{array}{l}\text { Mean NDVI change } \\
\text { visit } 1 \text { to visit } 2(\%)\end{array}$ \\
\hline Igurusi & 1190 & 19,796 & 1126 & 0.45 & -0.5 \\
\hline Mlowo & 1590 & 21,307 & 1956 & 0.45 & -2.5 \\
\hline Santylia & 2020 & 21,149 & 1802 & 0.57 & -0.2 \\
\hline Isongole & 2040 & 20,360 & 1889 & 0.57 & +2.9 \\
\hline
\end{tabular}

Table 2 Study population characteristics

\begin{tabular}{|c|c|c|c|c|c|}
\hline Site & Igurusi & Mlowo & Santylia & Isongole & Total \\
\hline Number of participants & 1126 & 1956 & 1802 & 1889 & 6773 \\
\hline Proportion of females; n/N (\%) & $593 / 1126(0.53)$ & $1027 / 1956(0.53)$ & $1001 / 1802(0.56)$ & $1045 / 1889(0.55)$ & $3666 / 6773(0.54)$ \\
\hline Median age at visit 1 in years (IQR) & $16.5(8.3 ; 36.1)$ & $14.9(7.8 ; 33.1)$ & $16.0(7.3 ; 34.2)$ & $16.0(7.8 ; 3.4)$ & $15.7(7.6 ; 34.7)$ \\
\hline \multicolumn{6}{|l|}{ Bed net ownership; n/N (\%) } \\
\hline Visit 1 & 1030/1126 (91.5\%) & 646/1956 (33.0\%) & $0 / 1802(0.0 \%)$ & $4 / 1889(0.2 \%)$ & $1680 / 6773(24.8 \%)$ \\
\hline Visit 2 & $998 / 1126(88.6 \%)$ & 796/1956 (40.7\%) & $32 / 1802(1.8 \%)$ & $31 / 1889(1.6 \%)$ & $1857 / 6773(27.4 \%)$ \\
\hline Visit 3 & $1022 / 1083(94.4 \%)$ & $1033 / 1845(56.0 \%)$ & $0 / 1622(0.0 \%)$ & $27 / 1762(1.5 \%)$ & $1082 / 6312(33.0 \%)$ \\
\hline $\begin{array}{l}\text { Change in bed net ownership (visit } 1 \text { to } \\
\text { visit 2) }\end{array}$ & $-3.1 \%$ & $+23.2 \%$ & NA & $+681 \%$ & $+10.5 \%$ \\
\hline \multicolumn{6}{|l|}{ Median SES-score (IQR) } \\
\hline Visit 1 & $0.01(-0.49 ; 0.64)$ & $0.13(-0.23 ; 0.63)$ & $-0.42(-0.75 ;-0.06)$ & $-0.36(-0.73 ; 0.00)$ & $-0.17(-0.58 ; 0.23)$ \\
\hline Visit 2 & $-0.02(-0.44 ; 0.54)$ & $0.16(-0.25 ; 0.71)$ & $-0.52(-0.82 ;-0.14)$ & $-0.42(-0.73 ; 0.14)$ & $-0.21(-0.62 ; 0.32)$ \\
\hline Visit 3 & $0.06(-0.54 ; 0.61)$ & $0.37(-0.13 ; 0.85)$ & $-0.51(-0.84 ;-0.02)$ & $-0.37(-0.80 ; 0.20)$ & $-0.14(-0.66 ; 0.45)$ \\
\hline \multicolumn{6}{|l|}{ P. falciparum RDT positivity; n/N (\%) } \\
\hline Visit 1 & $82 / 1126(7.3 \%)$ & $60 / 1956(3.1 \%)$ & $7 / 1802(0.4 \%)$ & $17 / 1889(0.9 \%)$ & $166 / 6773(2.5 \%)$ \\
\hline Visit 2 & $4 / 1126(0.4 \%)$ & $9 / 1956(0.5 \%)$ & $3 / 1802(0.2 \%)$ & $11 / 1889(0.6 \%)$ & $27 / 6773(0.4 \%)$ \\
\hline Visit 3 & $9 / 980(0.9 \%)$ & $4 / 1670(0.2 \%)$ & $1 / 1447(0.1 \%)$ & $0 / 1479(0.0 \%)$ & $14 / 5576(0.3 \%)$ \\
\hline Change in RDT + (visit 1 to visit 2) (\%) & -95.1 & -85.0 & -56.4 & -35.6 & -83.7 \\
\hline
\end{tabular}




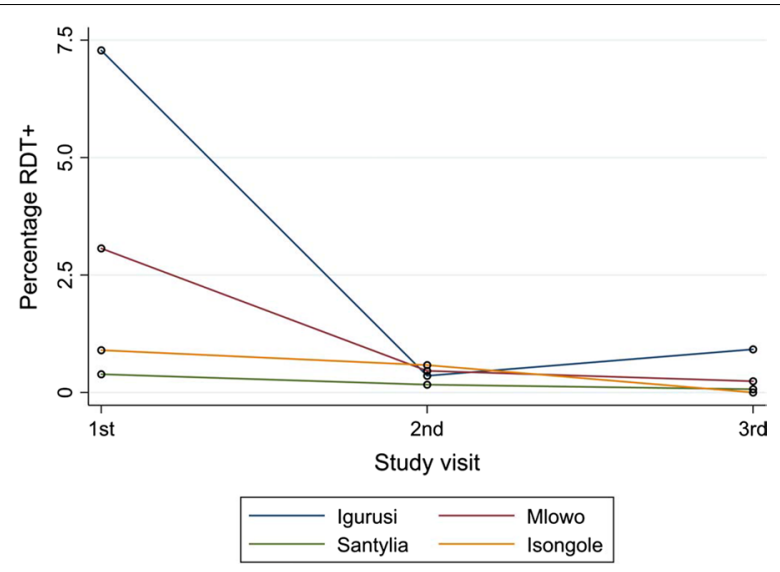

Fig. 2 Prevalence of $P$. falciparum positive RDT results over time by study site. ACT was introduced between visit 1 and visit 2 in each study site

2; six individuals were RDT positive at both visit 1 and 2 ; and 21 individuals were RDT negative at visit 1 and turned positive at visit 2; all remaining individuals were RDT negative at both visit 1 and visit 2 . At baseline, participants between 10 and 20 years of age were more often $P$. falciparum positive $(4.3 \%$; 69/1594) than all other age groups (Fig. 3). Furthermore, males across all age groups below 40 years had a higher prevalence of $P$. falciparum positivity $(3.0 \% ; 75 / 2525)$ than females of the same age group (2.4\%; 70/2973).

The proportion of bed net owners corresponded to the prevalence of malaria in the sites, with more bed net owners in the lower lying sites where malaria infection is common. As for the malaria high-prevalence site Igurusi, where also the proportion of bed net owners was high, individuals from households with bed nets showed lower prevalences of parasitaemia compared to individuals from households without bed nets located in the same site (Table 3 ). Figure 4 shows the proportion of bed net owners by site for all four sites over the three survey periods.

Change in bed net ownership between visits 1 and 2 and its potential influence on change in malaria prevalence was further analysed for Igurusi, the site with the highest malaria prevalence. Table 3 shows, that $P$. falciparum RDT positivity dropped substantially in all four groups, including participants who owned a bed net at the first visit but had lost it before the second visit.

Table 3 Change in bednet ownership and $P$. falciparum RDT positivity at visits 1 and 2 in Igurusi site

\begin{tabular}{lcc}
\hline Change in bed net ownership & $\begin{array}{l}\text { RDT + at visit 1 } \\
\text { n/N (\%) }\end{array}$ & $\begin{array}{l}\text { RDT + at visit 2 } \\
\text { n/N (\%) }\end{array}$ \\
\hline Lost bed net from visit 1 to visit 2 & $5 / 77(6.5 \%)$ & $0 / 77(0.0 \%)$ \\
No bed net at both visits 1 and 2 & $15 / 51(29.4 \%)$ & $0 / 51(0.0 \%)$ \\
$\begin{array}{l}\text { Bed net at both visits 1 and 2 } \\
\begin{array}{l}\text { Acquired bed net from visit 1 to } \\
\text { visit 2 }\end{array}\end{array}$ & $58 / 953(6.1 \%)$ & $4 / 953(0.4 \%)$ \\
\hline
\end{tabular}

This comparison of change in bed net ownership and $P$. falciparum infection is provided for Igurusi only, the location with the highest overall prevalence of $P$. falciparum infection at visit 1

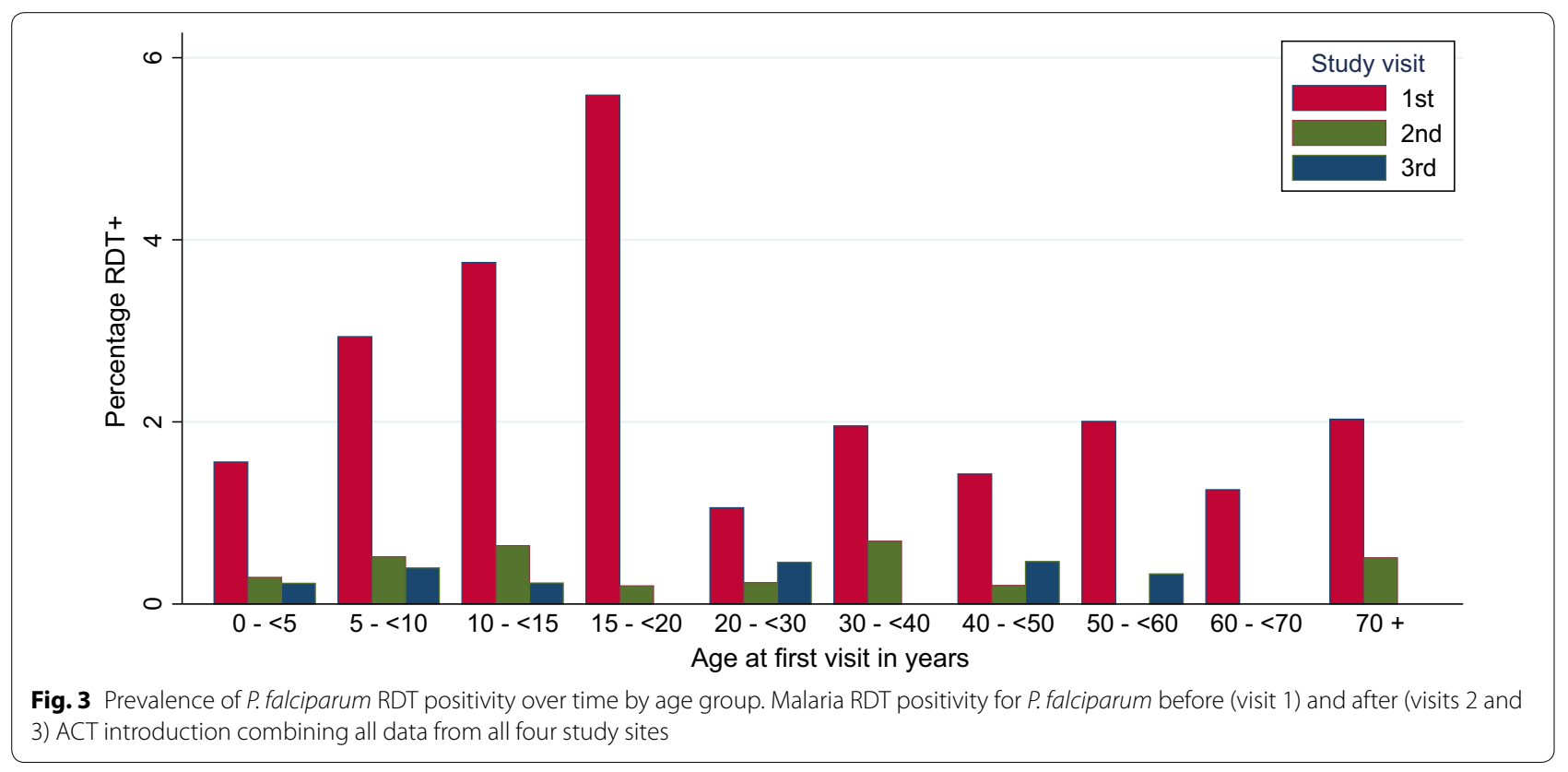




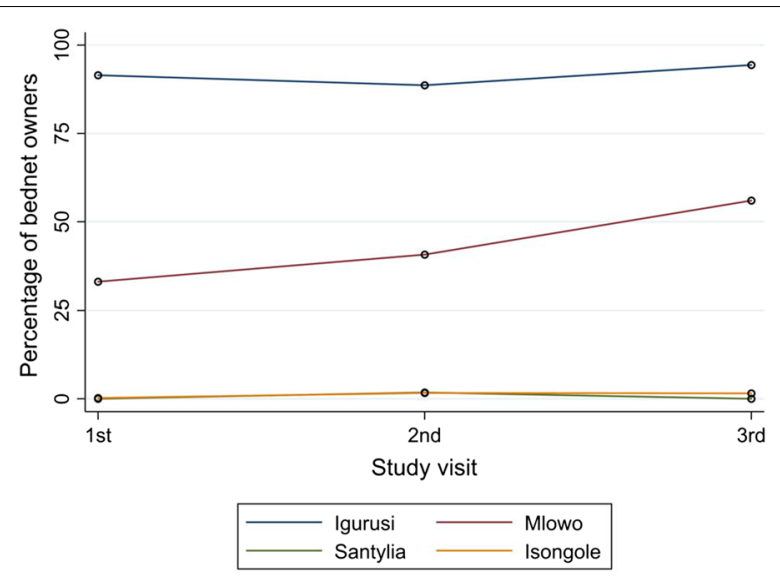

Fig. 4 Proportions of bed net owners over time

\section{Multivariable analysis}

In total 6769 participants with $P$. falciparum RDT results for the first and second visit were included in the two uni- and multivariable mixed effects logistic regression models. The first model aims to identify factors that might influence $P$. falciparum infection at the first visit. The second model examines the influence that potential change of the above identified factors between the visits might have on the decline in malaria between visits. The first model revealed significantly higher odds of $P$. falciparum infection for participants between 5 and 10 (OR
2.05, 95\% CI 1.32-3.17) and between 10 and 20 years of age (OR $=3.04,95 \%$ CI 2.14-4.32) compared to children below 5 years. Bed net ownership, and SES were negatively associated with RDT positivity whereas NDVI showed a positive association. These associations were relatively strong, but significant only in univariable, and not in multivariable analysis (Table 4).

Nevertheless, these factors were included into the second model, where the influence of changes in bed net ownership, NDVI and SES on the observed change in malaria prevalence between the first and second visit were assessed, again using uni- and multivariable mixed effects logistic regression models, but this time with loss of $P$. falciparum infection, i.e. change in RDT status from positive to negative as the outcome.

Although unchanged between visits, age at visit 1 and gender were included into the models for loss of $P$. falciparum infection as potential confounders (Table 5). The multivariable model showed significantly higher odds of changing to negative for those groups who also had higher odds of infection at visit 1: male participants and participants between 5 and $10(\mathrm{OR}=1.94,95 \% \mathrm{CI} 1.29$ $2.91)$ and between 10 and 20 years of age $(\mathrm{OR}=2.98,95 \%$ CI 2.10-4.23) compared to children below 5 years, had higher odds of changing to $P$. falciparum negative. Participants who lost or obtained a bed net between surveys both changed more often to negative than participants with unchanged bed net status (3.79 and $2.84 \%$ versus

Table 4 Uni- and multivariable mixed effects logistic regression model for $P$. falciparum RDT positivity at visit 1

\begin{tabular}{|c|c|c|c|c|c|c|c|c|c|}
\hline \multirow[t]{2}{*}{ Covariate } & \multirow[t]{2}{*}{$\mathbf{N}$} & \multirow[t]{2}{*}{ N-pos. } & \multirow[t]{2}{*}{ \%-pos. } & \multicolumn{3}{|c|}{ Univariable } & \multicolumn{3}{|c|}{ Multivariable } \\
\hline & & & & OR & $95 \% \mathrm{Cl}$ & $p$ value & OR & $95 \% \mathrm{Cl}$ & $p$ value \\
\hline \multicolumn{10}{|l|}{ Gender } \\
\hline Female $^{a}$ & 3663 & 84 & 2.29 & 1.00 & - to - & - & 1.00 & - to - & - \\
\hline Male & 3106 & 82 & 2.64 & 1.14 & (0.99 to 1.31$)$ & 0.0747 & 1.10 & (0.95 to 1.26$)$ & 0.1929 \\
\hline \multicolumn{10}{|l|}{ Age at Srv1 (years) } \\
\hline $0-<5^{\mathrm{a}}$ & 1026 & 16 & 1.56 & 1.00 & - to - & - & 1.00 & - to - & - \\
\hline $5-<10$ & 1158 & 34 & 2.94 & 2.07 & (1.35 to 3.17$)$ & 0.0009 & 2.05 & (1.32 to 3.17$)$ & 0.0013 \\
\hline $10-<20$ & 1592 & 69 & 4.33 & 3.10 & (2.20 to 4.37$)$ & $<0.0001$ & 3.04 & (2.14 to 4.32$)$ & $<0.0001$ \\
\hline $20-<40$ & 1720 & 26 & 1.51 & 1.03 & (0.49 to 2.18$)$ & 0.9356 & 1.06 & (0.50 to 2.22$)$ & 0.8802 \\
\hline$\geq 40$ & 1273 & 21 & 1.65 & 1.09 & (0.48 to 2.43 ) & 0.8426 & 0.99 & (0.42 to 2.32) & 0.9808 \\
\hline \multicolumn{10}{|l|}{ Bed net owned } \\
\hline $\mathrm{No}^{\mathrm{a}}$ & 5089 & 89 & 1.75 & 1.00 & - to - & - & 1.00 & - to - & - \\
\hline Yes & 1680 & 77 & 4.58 & 0.44 & (0.22 to 0.92$)$ & 0.0281 & 0.56 & (0.18 to 1.73$)$ & 0.3139 \\
\hline \multicolumn{10}{|l|}{ SES-score } \\
\hline Per unit & - & - & - & 0.72 & (0.64 to 0.81$)$ & $<0.0001$ & 0.81 & (0.65 to 1.00$)$ & 0.0515 \\
\hline \multicolumn{10}{|l|}{ NDVI } \\
\hline Per 0.1 NDVI units & - & - & - & 2.20 & (1.01 to 4.82 ) & 0.0479 & 1.83 & (0.81 to 4.17$)$ & 0.1490 \\
\hline
\end{tabular}

Using robust variance estimates and including site and household as random effects to adjust for clustering, $\mathrm{N}=6769$

$\mathrm{N}$ number of observations, $\mathrm{N}$-pos. number of positives, $\%$-pos. percent positive, OR odds ratio, $95 \% \mathrm{Cl} 95 \%$ confidence interval

a Reference stratum 
Table 5 Uni- and multivariable mixed effects logistic regression models to assess the potential influence of changes in bed net ownership, SES and NDVI between visit 1 and visit 2 on change of $P$. falciparum RDT result from positive at visit 1 to negative at visit 2

\begin{tabular}{|c|c|c|c|c|c|c|c|c|c|}
\hline \multirow[t]{2}{*}{ Covariate } & \multirow[t]{2}{*}{$\mathbf{N}$} & \multirow[t]{2}{*}{ N-chgd. } & \multirow[t]{2}{*}{ \%-chgd. } & \multicolumn{3}{|c|}{ Univariable } & \multicolumn{3}{|c|}{ Multivariable } \\
\hline & & & & OR & $95 \% \mathrm{Cl}$ & $p$ value & OR & $95 \% \mathrm{Cl}$ & p value \\
\hline \multicolumn{10}{|l|}{ Gender } \\
\hline Female $^{a}$ & 3663 & 80 & 2.18 & 1.00 & - to - & - & 1.00 & - to - & - \\
\hline Male & 3106 & 80 & 2.58 & 1.17 & (0.97 to 1.41$)$ & 0.0950 & 1.12 & (0.94 to 1.35$)$ & 0.2042 \\
\hline \multicolumn{10}{|l|}{ Age at visit 1, years } \\
\hline $0-<5^{\mathrm{a}}$ & 1026 & 16 & 1.56 & 1.00 & - to - & - & 1.00 & - to - & - \\
\hline $5-<10$ & 1158 & 32 & 2.76 & 1.95 & (1.31 to 2.88$)$ & 0.0009 & 1.94 & (1.29 to 2.91$)$ & 0.0013 \\
\hline $10-<20$ & 1592 & 67 & 4.21 & 3.00 & (2.12 to 4.26$)$ & $<0.0001$ & 2.98 & (2.10 to 4.23$)$ & $<0.0001$ \\
\hline $20-<40$ & 1720 & 25 & 1.45 & 0.98 & (0.49 to 1.99$)$ & 0.9658 & 0.99 & (0.47 to 2.07$)$ & 0.9823 \\
\hline$\geq 40$ & 1273 & 20 & 1.57 & 1.02 & (0.50 to 2.07$)$ & 0.9588 & 1.00 & (0.49 to 2.03$)$ & 0.9947 \\
\hline \multicolumn{10}{|c|}{ Change in bed net ownership } \\
\hline Lost net & 211 & 8 & 3.79 & 0.74 & (0.67 to 0.82$)$ & $<0.0001$ & 0.76 & $(0.57$ to 1.02$)$ & 0.0669 \\
\hline No change ${ }^{a}$ & 6170 & 141 & 2.29 & 1.00 & - to - & - & 1.00 & - to - & - \\
\hline Received net & 388 & 11 & 2.84 & 0.99 & (0.49 to 1.98$)$ & 0.9732 & 1.04 & (0.48 to 2.24$)$ & 0.9209 \\
\hline \multicolumn{10}{|c|}{ Change in SES score } \\
\hline (Per unit) & - & - & - & 0.93 & (0.74 to 1.17$)$ & 0.5614 & 0.96 & $(0.70$ to 1.31$)$ & 0.7984 \\
\hline \multicolumn{10}{|c|}{ Relative change in NDVI } \\
\hline Per $1 \%$ change & - & - & - & 1.10 & (1.00 to 1.21$)$ & 0.0565 & 1.10 & (0.99 to 1.22$)$ & 0.0756 \\
\hline
\end{tabular}

Using robust variance estimates and including site and household as random effects to adjust for clustering, $N=6769$

$N$ number of observations, $N$-chgd. and \%-chgd. number and percentage of participants who changed from $P$. falciparum positive at visit 1 to negative at visit $2, O R$ odds ratio, 95\% Cl 95\% confidence interval

a Reference stratum

$2.29 \%$ respectively). However, when adjusting for site and household in the mixed effects models, the odds of losing infection were considerably lower for participants who lost their bed net compared to those with unchanged bed net ownership in both the uni- $(\mathrm{OR}=0.74,95 \% \mathrm{CI}$ $0.67-0.82)$ and multivariable analysis $(\mathrm{OR}=0.76,95 \% \mathrm{CI}$ 0.57-1.02), whereas the odds for those who obtained a bed net were nearly the same as for those where bed net ownership did not change.

Change in SES showed a very weak and non-significant negative association with loss of malaria infection. Change in NDVI showed a stronger but non-significant positive association, in both the uni- and multivariable analysis ( $\mathrm{OR}=1.10,95 \% \mathrm{CI} 0.99-1.22)$, meaning that loss of $P$. falciparum infection between visits was higher when NDVI increased, which is counterintuitive.

\section{Discussion}

In the EMINI cohort, replacement of sulfadoxine/ pyrimethamine (SP) by ACT as first-line therapy in the years 2006 and 2007 resulted in a significant and relevant reduction of $P$. falciparum prevalence. In this study, malaria status was determined by RDT, which reflects common procedures in health care systems in malaria endemic countries. This strategy is somewhat hampered by limited sensitivity and specificity, which may be particularly challenging the positive predictive value of any given RDT in a setting where malaria prevalence has been already substantially reduced. Between baseline (visit 1) and the first follow up (visit 2) an overall 83.7\% reduction in $P$. falciparum prevalence could be shown. The effect was sustained and even consolidated afterwards, resulting in an overall prevalence of $P$. falciparum RDT positivity of only $0.3 \%$ at visit 3 compared to $2.5 \%$ at baseline. Similarly promising findings have been reported from other countries that are endemic for malaria [6]. A reduced prevalence of parasitaemia is a key factor in reducing transmission, in particular the reduced prevalence of gametocyte carriers, which is achieved through the gametocidal activity of ACT. However, some studies still suggest high prevalences of Plasmodium infection in the general population after ACT implementation as a continued driver for sustained transmission [20-22].

The crude prevalence of Plasmodium infection differed significantly between sites, with higher prevalences in the lower altitude sites of Igurusi and Mlowo, compared to the high altitude sites Santylia and Isongole. The mixed effects logistic regression model found that age between 
5 and 20 years was a significant predictor for a higher risk of $P$. falciparum infection, an age dependent distribution of prevalence, which has been described in other studies $[4,6]$. It appears that younger children are better protected, for example by the use of the existing bed nets, whereas adolescents in the age range from 10 to 20 years seem to be particularly exposed due to staying outside homes also during evening and night hours with greatest risk of transmission. At baseline, males were slightly more often $P$. falciparum infected than females, as has been shown in other studies [4]. The model further showed that owning a bed net and SES are negatively, and NDVI values at the place of residence are positively associated with $P$. falciparum infection, although these associations were significant only in univariable analysis (Table 4).

When looking at the change of $P$. falciparum infection status from visit 1 to visit 2 , we found that age between 5 and 20 years was the only factor that was significantly associated with a change from $P$. falciparum positive to negative between the two visits. Since this age group had the highest malaria prevalence at baseline, the potential for changing to malaria negative is also highest, which is corroborated by our findings. The same is true for the non-significant association of male gender with loss of malaria infection. Changing SES only showed a weak association with loss of malaria infection.

The odds for switching to RDT negative for participants who obtained a bed net between surveys and for those whose bed net ownership status did not change are very similar. The large majority of individuals had a stable bed net ownership status, that is: keeping their bed net or staying without one, which in turn depended on the background prevalence of $P$. falciparum infection. The group that received a bed net remained small, only 11 individuals in this group showed a switch from infection status positive at visit 1 to negative at visit 2 (Table 5). This shows that a higher bed net coverage is not the driving factor for the observed drop in P. falciparum infection after ACT introduction, at least in this setting with the reported baseline coverage of bed nets. It has to be pointed out, that the variable of bed net ownership bears its limitations, as actual use of the bed net is not assessed.

The association of increasing NDVI with switching to malaria negative, which is close to significant, is counterintuitive. Increasing NDVI, as a marker of increasing humidity, should rather have been associated with a lower decrease in malaria infection. Thus this result is hard to explain, but at least shows that changes in vegetation do not explain the drop of malaria prevalence between visit 1 and visit 2 . It has to be pointed out, that on a site level the differences in altitude between the sites will have played a greater role than NDVI. However,
NDVI values were determined on a household level, and here neither significant association nor intuitive trend could be established.

A challenge in malaria control may be seen in areas with inherently rather low malaria prevalence, such as the Mbeya highlands. Here household decision makers may be tempted to neglect protective measures such as the use of ITNs for their children, owed to a rarer occurrence of malaria. This study shows a relevant increase in bed net ownership only for Mlowo, one of the lower altitude sites with a higher prevalence of malaria infection. The other three sites did not show a consistent change in bed net ownership. However, due to an incomplete adaptive immunity in this altogether hypoendemic situation and inbound mobility of potentially parasitaemic individuals from hyperendemic areas, the population of these hypoendemic regions remains at particular risk for severe courses of malaria.

The model for baseline malaria infection indicated that higher SES is protective against malaria infection. However, in the regression model on change in infection status, no conclusive effect of SES change on switching to malaria negative was found.

The crude average NDVI values at baseline were similar for the two low lying sites, and for the two higher lying sites respectively. The reduction of NDVI-and thus vegetation-between visit 1 and visit 2 likely indicates decreased rainfall for Igurusi and Mlowo, the sites with the largest absolute and relative reductions in malaria prevalence. Only Isongole, the site at highest altitude of $2040 \mathrm{~m}$, showed an increase in NDVI; this site, however, contributed least to the overall reduction in malaria prevalence. However, when adjusting for site and household in the models on change in P. falciparum status (Table 5) this correlation between decreasing NDVI and increased odds of losing $P$. falciparum positivity could not be ascertained at the individual level.

The most important limitation of this study is the lack of a control group which would have enabled us to directly compare the impact of ACT treatment on malaria with that of SP treatment, the previous standard of care. Instead the analysis relies on a before/after comparison, which might have been confounded by other factors that changed between the two visits. In order to overcome this, the most important factors that were associated with malaria at baseline were identified, and an analysis was performed in order to examine whether their change could explain the observed drop in P. falciparum prevalence, which was not the case. Furthermore, to the knowledge of the authors, no additional systematic malaria control activities such as indoor residual spraying (IRS) were implemented in the region during the study period. Although other uncontrolled factors also might 
have played a role in this, the change in first-line treatment as corroborated through this study seems to sufficiently explain the observed change in $P$. falciparum prevalence.

A selection bias imposed by the exclusion of participants that had missed consecutive visits cannot be ruled out.

The validity of NDVI as a proxy indicator of humidity and, therefore, of favourable conditions for vector habitats is limited. NDVI has shown to correlate with rainfall only in defined lower ranges of annual rainfall. Mbeya region with about $800 \mathrm{~mm}$ annual rainfall is already exceeding the upper limit of linear correlation. In addition, many other factors such as lag time and fine scale variability over time were not considered [23, 24]. Another weakness are uncontrolled influences of urban areas on NDVI values, as sealed surfaces and construction activities disturb the correlation between NDVI and potential presence of vector habitat.

\section{Conclusions}

The introduction of ACT in the Mbeya Region coincides with a relevant reduction in malaria prevalence within a very short period of time. Since this decrease is not explained by other factors included in the presented analyses, the investigators believe that it reflects the effect of switching to ACT as first-line malaria therapy. Lower prevalence of $P$. falciparum infection implies a lower transmission and, therefore, lower morbidity and mortality in vulnerable age groups, which comprise children and adolescents between 5 and 20 years of age. The study area is by now considered a low risk area for malaria transmission. At the same time the local health authorities have adopted an improved approach of antimicrobial stewardship with the introduction of RDTs and by reserving anti-malarial treatment for patients with a positive RDT result. In addition, this development improves the adequate use of antibiotics, which are not administered as a fixed combination with anti-malarials anymore. These are important prerequisites for avoiding development of resistances against first line anti-malarials currently in use.

\footnotetext{
Abbreviations

ACT: artemisinin-based combination therapy; EMINI: Establishment of the infrastructure for the Evaluation and Monitoring of the Impact of New Interventions; HRP2: histidine-rich protein 2; IRS: indoor residual spraying; ITN: insecticide-treated net; MMRC: Mbeya Medical Research Center; NDVI: Normalized Difference Vegetation Index; RDT: rapid diagnostic testing; SES: socio-economic status; SP: sulfadoxine/pyrimethamine.
}

\section{Authors' contributions}

$\mathrm{MH}$ and LM secured funding and designed the EMINI study together with ES. IK, PC, WA, WM and MG were responsible for coordination of field work, data collection, sample collection and laboratory analysis. GF and ES analysed the data with input from NBR and ENN. GF and ES wrote the initial draft of the manuscript. All authors read and approved the final manuscript.

\section{Author details}

${ }^{1}$ Division of Infectious Diseases and Tropical Medicine, University Hospital, LMU Munich, Leopoldstr. 5, 80802 Munich, Germany. ${ }^{2}$ German Center for Infection Research (DZIF), Partner Site Munich, Leopoldstr. 5, 80802 Munich, Germany. ${ }^{3}$ National Institute of Medical Research-Mbeya Medical Research Centre, P.O. Box 2410, Hospital Hill Road, Mbeya, Tanzania.

\section{Acknowledgements}

The authors would like to thank all participants in the Tanzanian study sites for their interest and support for the cohort study. Equal gratitude goes to the Tanzanian authorities for supporting this research in the Mbeya Region.

\section{Competing interests}

The authors declare that they have no competing interests.

\section{Availability of data and materials}

The datasets generated and analysed during the current study are not publicly available due to confidentiality concerns (GIS positions of the homes are an integral part of the dataset), but are available from the corresponding author on reasonable request.

\section{Consent for publication}

Not applicable.

\section{Ethics approval and consent to participate}

All participants provided written informed consent prior to inclusion into this study, with legal guardians consenting for minors below 18 years. Children between 12 and 18 years additionally provided written assent to participate in the study. Approval of the cohort study within the EMINI framework has been obtained from the Tanzanian National Institute for Medical Research and the institutional ethics committee of the University of Munich, Germany.

\section{Funding}

The EMINI study was funded by the European Union (SANTE/2004/078-545 and SANTE/2006/129-931). The funding agency had no role in study design, data collection, data analysis and publication of the findings.

\section{Publisher's Note}

Springer Nature remains neutral with regard to jurisdictional claims in published maps and institutional affiliations.

Received: 21 March 2018 Accepted: 13 June 2018

Published online: 26 June 2018

\section{References}

1. National Bureau of Statistics. Tanzania in figures 2012. Dar es Salaam: Ministry of Finance, United Republic of Tanzania; 2013.

2. United Republic of Tanzania. Human development indicators. http://hdr. undp.org/en/countries/profiles/TZA. Accessed 5 Sept 2017.

3. WHO. United Republic of Tanzania: WHO statistical profile. Geneva: World Health Organization; 2015.

4. Mazigo HD, Rumisha SF, Chiduo MG, Bwana VM, Mboera LEG. Malaria among rice farming communities in Kilangali village, Kilosa district, Central Tanzania: prevalence, intensity and associated factors. Infect Dis Poverty. 2017;6:101

5. WHO. World malaria report 2012. Geneva: World Health Organization 2012.

6. Khatib RA, Skarbinski J, Njau JD, Goodman CA, Elling BF, Kahigwa E, et al. Routine delivery of artemisinin-based combination treatment at fixed health facilities reduces malaria prevalence in Tanzania: an observational study. Malar J. 2012;11:140.

7. Masaninga F, Chanda E, Chanda-Kapata P, Hamainza B, Masendu HT, Kamuliwo $\mathrm{M}$, et al. Review of the malaria epidemiology and trends in Zambia. Asian Pac J Trop Biomed. 2013;3:89-94. 
8. Bhattarai A, Ali AS, Kachur SP, Martensson A, Abbas AK, Khatib R, et al. Impact of artemisinin-based combination therapy and insecticidetreated nets on malaria burden in Zanzibar. PLoS Med. 2007;4:e309.

9. Willilo RA, Molteni F, Mandike R, Mugalura FE, Mutafungwa A, Thadeo A, et al. Pregnant women and infants as sentinel populations to monitor prevalence of malaria: results of pilot study in Lake Zone of Tanzania. Malar J. 2016;15:392.

10. Kajeguka DC, Kaaya RD, Mwakalinga S, Ndossi R, Ndaro A, Chilongola JO, et al. Prevalence of dengue and chikungunya virus infections in northeastern Tanzania: a cross sectional study among participants presenting with malaria-like symptoms. BMC Infect Dis. 2016;16:183.

11. Briggs MA, Kalolella A, Bruxvoort K, Wiegand R, Lopez G, Festo C, et al. Prevalence of malaria parasitemia and purchase of artemisinin-based combination therapies (ACTs) among drug shop clients in two regions in Tanzania with ACT subsidies. PLoS ONE. 2014;9:e94074.

12. World Bank Group. http://sdwebx.worldbank.org/climateportal/index .cfm?page=country_historical_climate\&ThisCCode=TZA. Accessed 5 Sept 2017.

13. Farr TG, Rosen PA, Caro E, Crippen R, Duren R, Hensley S, et al. The shuttle radar topography mission. Rev Geophys. 2007:45:RG2004

14. Rodríguez E, Morris CS, Belz JE, Chapin EC, Martin JM, Daffer W, et al. An assessment of the SRTM topographic products. Technical Report JPL, D-31639. Pasadena: Jet Propulsion Laboratory; 2005. p. 143.

15. Filmer D, Pritchett LH. Estimating wealth effects without expenditure data - or tears: an application to educational enrollments in states of India. Demography. 2001;38:115-32.

16. Kolenikov SA, Angeles G. Socioeconomic status measurement with discrete proxy variables: is principal component analysis a reliable answer? Review of Income and Wealth. 2009:55:128-65.
17. NASA Land Processes Distributed Active Archive Center (LP DAAC): MODIS 11A2. USGS/Earth Resources Observation and Science (EROS) Center, Sioux Falls, South Dakota; 2001.

18. NASA Land Processes Distributed Active Archive Center (LP DAAC): MODIS 13Q1. Resources Observation and Science (EROS) Center, Sioux Falls, South Dakota; 2001.

19. NASA. http://earthobservatory.nasa.gov/Features/MeasuringVegetation/ printall.php. Accessed 5 Sept 2017.

20. Jenkins R, Omollo R, Ongecha M, Sifuna P, Othieno C, Ongeri L, et al. Prevalence of malaria parasites in adults and its determinants in malaria endemic area of Kisumu County, Kenya. Malar J. 2015;14:263.

21. De Beaudrap P, Nabasumba C, Grandesso F, Turyakira E, Schramm B, Boum $Y$ 2nd, et al. Heterogeneous decrease in malaria prevalence in children over a six-year period in south-western Uganda. Malar J. 2011;10:132.

22. Alves FP, Gil LH, Marrelli MT, Ribolla PE, Camargo EP, Da Silva LH. Asymptomatic carriers of Plasmodium spp. as infection source for malaria vector mosquitoes in the Brazilian Amazon. J Med Entomol. 2005:42:777-9.

23. Espinosa LAD, Posadas AN, Carbajal M, Quiroz R. Multifractal downscaling of rainfall using normalized difference vegetation index (NDVI) in the Andes Plateau. PLoS ONE. 2017;12:e0168982.

24. Martiny N, Camberlin P, Richard Y, Philippon N. Compared regimes of $\mathrm{NDVI}$ and rainfall in semi-arid regions of Africa. Int J Remote Sensing. 2006;27:5201-23.
Ready to submit your research? Choose BMC and benefit from:

- fast, convenient online submission

- thorough peer review by experienced researchers in your field

- rapid publication on acceptance

- support for research data, including large and complex data types

- gold Open Access which fosters wider collaboration and increased citations

- maximum visibility for your research: over 100M website views per year

At BMC, research is always in progress.

Learn more biomedcentral.com/submissions 